\title{
Desigualdades geográficas en las cargas de trabajo por dentista en España entre 1987 y 1997. Cargas de trabajo por dentista

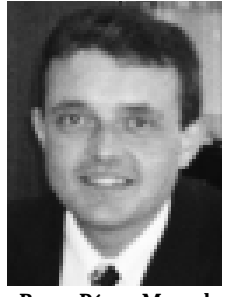 \\ Bravo-Pérez, Manuel
}

\section{Inequalities in the workload per dentist in Spain from 1987 to 1997. Workload per dentist}

Bravo-Pérez, Manuel*

Doctor y Profesor Titular de Odontología Preventiva y Comunitaria. Departamento de Estomatología. Facultad de Odontología. Universidad de Granada.

Financiación: Estudio parcialmente financiado por el Grupo de Investigación «Salud Pública Oral y Gerodontología (código CTS503)», de la Junta de Andalucía.

\begin{abstract}
Resumen: Introducción (fundamento y objetivo): durante las dos últimas décadas, la Odontología española asistió a un fuerte incremento del número de profesionales, habiéndose demostrado una reducción del 42,0\% en las cargas privadas por dentista entre 1987 y 1997. El objetivo ha sido comparar esta pérdida de cargas de trabajo entre Comunidades Autónomas (CCAA). Método: se utilizaron los censos poblacionales y de dentistas, así como las Encuestas Nacionales de Salud. Se calculó el porcentaje de población que visitó al dentista en los tres meses previos a cada encuesta, y de ellos, el porcentaje cuya última visita fue privada. El total de visitas realizadas por esa población sirvió para estimar el total de visitas privadas en cada CCAA durante tres meses, que se dividió por el número de dentistas. Resultados y conclusiones: tanto en 1987 como en 1997 hay diferencias significativas en las cargas de trabajo por dentista según CCAA, si bien no en la reducción de las mismas.
\end{abstract}

Palabras Clave: Dentista, Utilización odontológica, Cargas de trabajo, España.

Abstract: Introduction and purpose: During the last two decades Spanish Dentistry has witnessed a great increase in the number of dentists, with a $42.0 \%$ reduction in the private workload per dentist between 1987 and 1997. The aim of this study was to compare the reduction in workload between the different Spanish Autonomous Communities (CCAA). Methods: Data were taken from censuses of the population and of dental practitioners, as well as from the Spanish National Health Interview Surveys. The percentage of persons who had visited the dentist in the three months prior to the survey was calculated, as well as the percentage of persons of this last group whose last visit had been to a private practitioner. The total number of visits made by this population was taken as the estimate of total private visits in each CCAA during three months. Total private activity was then divided by the number of dentists. Results and Conclusiones: Both in 1987 and 1997 there are significant differences in the workload per dentist depending on the CCAA, although not in the workload reduction during that period of time.

Key Words: Dentist, Dental utilisation, Workload, Spain.

\begin{tabular}{ccc}
\hline Fecha recepción & Fecha última revisión & Fecha aceptación \\
$23-02-2004$ & $15-03-2004$ & $30-03-2004$ \\
\hline
\end{tabular}

BIBUD [1138-123X (2004)9:3; mayo-junio 257-360]

Bravo-Pérez M. Desigualdades geográficas en las cargas de trabajo por dentista en España entre 1987 y 1997. Cargas de trabajo por dentista. RCOE 2004;9(3):277-284. 


\section{Introducción}

La Profesión Odontológica española está sufriendo desde mediados de los años 80 un incremento del número de sus miembros, descontrolado, y sin similitud con el resto de países de la $\mathrm{UE}^{1,2^{2 *}, 3^{*}}$. De acuerdo con las necesidades objetivas de tratamiento odontológico, y utilizando el programa informático específico JWG6 de la FDI/OMS, que permite calcular el número de dentistas necesarios, se ha estimado, para 1993, una ratio óptima en España de 2350-2800 habitantes/ dentista ${ }^{4}$, y entre 2700-3200 habitantes/dentista en $2000^{5}$, cifra ya superada, particularmente en las capitales.

La distribución geográfica de los profesionales ha sido descrita e indica una situación desfavorable para las zonas rurales ${ }^{1}$, posiblemente relacionada con el perfil privado de la profesión $^{2 * * 6}$. Aunque se ha informado de una reducción de las cargas de trabajo privadas por dentista de un $42,0 \%$ entre 1987 y $1997^{2 *}$, no se ha analizado si hay diferencias geográficas tanto en la demanda de asistencia odontológica, como en las cargas de trabajo por dentista.

Los objetivos a partir de los datos de las Encuestas Nacionales de Salud (ENSs) (Ministerio de Sanidad y Consumo) disponibles, de los datos poblacionales del INE (Instituto Nacional de Estadística) y de los censos de dentistas (Consejo General Colegios de Odontólogos y Estomatólogos, e INE), han sido evaluar la influencia de la Comunidad Autónoma (CCAA) en la demanda de asistencia odontológica y en las cargas de trabajo privado por dentista en España entre 1987 y
1997, periodo de máximo crecimiento del número de profesionales.

\section{Material y método}

\section{Encuestas Nacionales de Salud}

El estudio está basado en las ENSs de $1987^{7}$ (ENS87, $\mathrm{n}=39751$ ), y $1997^{\circ}$ (ENS97, $\mathrm{n}=8383$ ). Las ENSs fueron dirigidas por el Ministerio de Sanidad, y desarrolladas por el Centro de Investigaciones Sociológicas. Se basan en muestras representativas de la población española, excluyendo a las ciudades autónomas de Ceuta y Melilla, y a los residentes en instituciones, que representaban el $0,7 \%$ de la población en 1987 .

Las ENSs son cuestionarios realizados a domicilio por entrevistadores entrenados, y contienen preguntas sobre morbilidad, estado de salud y uso de servicios sanitarios. Los cuestionarios infantiles ( $<16$ años) fueron respondidos por la madre, padre, o familiar cercano al escolar. La ENS87 se llevó a cabo fundamentalmente en julio de 1987, y la ENS97 durante todo el año, para eliminar el posible efecto estacional.

Junto a las variables de diseño muestral (región, provincia, etc.), se utilizaron algunas cuestiones específicas para este estudio. En primer lugar, se les preguntó si habían realizado alguna visita al dentista en los últimos tres meses. A los que respondieron que sí, se les preguntó cuántas veces, y el tipo de dentista (Seguridad Social, ayuntamiento-beneficencia, seguro médico, privado u otros). Para el objetivo de este estudio, «Seguridad Social» y «ayuntamiento-beneficencia» se clasificación como dentista público, y «seguro médico» y «privado» como dentista privado; «otros» se consideró desconocido.

\section{Población y número de dentistas (censos)}

Los datos de población y de dentistas se tomaron del INE, excluyendo Ceuta y Melilla (tabla 1). Los datos fueron ajustados por interpolación lineal para las fechas representativas de cada ENSs. El listado de dentistas sólo incluye odontólogos o médicos estomatólogos colegiados con ejercicio profesional. Todos los dentistas se consideraron que independientemente de que fueran empleados en el sector público, también ejercían como dentistas privados. Esta aproximación ha sido demostrada válida para el periodo de estudio ${ }^{2 * *}$.

\section{Análisis estadístico}

El análisis ha considerado el muestreo complejo de las ENS, y además se ajustaron los pesos para los no respondientes. Se utilizó el programa estadístico SUDAAN ${ }^{9}$, introduciendo el método WOR (sin reemplazamiento) como tipo de diseño muestral. Para cada año, y utilizando los procedimientos DESCRIPT (variables cuantitativas) y CROSSTAB (porcentajes) en SUDAAN, se calculó el porcentaje de población que visitó al dentista en los últimos tres meses, y a continuación el porcentaje de últimas visitas que fueron privadas. El número total que esa población realizó, se tomó como la cantidad total de visitas odontológicas privadas en España en tres meses.

A continuación, esas cantidades fueron divididas por el número de dentistas, lo que permitió la estimación de las cargas de trabajo en tres meses. La 


\begin{tabular}{|c|c|c|c|c|c|c|c|c|c|}
\hline \multicolumn{10}{|c|}{ Tabla l' Población y número de dentist } \\
\hline \multirow{3}{*}{$\begin{array}{l}\text { Variable } \\
\text { Todos }\end{array}$} & \multicolumn{3}{|c|}{ Población (en miles) } & \multicolumn{3}{|c|}{ № de dentistas } & \multicolumn{3}{|c|}{ Ratio hab./ dentista (miles) } \\
\hline & \multirow{2}{*}{$\begin{array}{l}1987 \\
38679 \\
\end{array}$} & \multirow{2}{*}{$\frac{1997}{39662}$} & \multirow{2}{*}{$\frac{\text { cambio \% }}{2.5}$} & \multirow{2}{*}{1987} & \multicolumn{2}{|c|}{1997 cambio \% } & \multirow{2}{*}{$\begin{array}{l}1987 \\
6070 \\
\end{array}$} & \multicolumn{2}{|c|}{1997 cambio \% } \\
\hline & & & & & 15045 & 136.1 & & 2636 & -56.6 \\
\hline Comunidad Autónoma & & & & & & & & & \\
\hline Andalucía & 6875 & 7245 & 5,4 & 831 & 2406 & 189,7 & 8275 & 3011 & $-63,6$ \\
\hline Aragón & 1189 & 1186 & $-0,3$ & 230 & 460 & 99,7 & 5166 & 2580 & $-50,1$ \\
\hline Baleares & 713 & 769 & 7,9 & 120 & 330 & 174,5 & 5930 & 2331 & $-60,7$ \\
\hline Canarias & 1499 & 1612 & 7,6 & 189 & 497 & 163,5 & 7941 & 3241 & $-59,2$ \\
\hline Castilla León & 2599 & 2503 & $-3,7$ & 331 & 797 & 140,6 & 7848 & 3142 & $-60,0$ \\
\hline Cataluña & 5986 & 6129 & 2,4 & 1103 & 2339 & 112,0 & 5246 & 2621 & $-51,7$ \\
\hline Extremadura & 1096 & 1070 & $-2,3$ & 72 & 213 & 195,8 & 15208 & 5022 & $-67,0$ \\
\hline Galicia & 2871 & 2738 & $-4,6$ & 306 & 1026 & 234,7 & 9370 & 2670 & $-71,5$ \\
\hline Navarra & 519 & 523 & 0,9 & 96 & 221 & 130,1 & 5388 & 2362 & $-56,2$ \\
\hline Madrid & 4823 & 5065 & 5,0 & 1371 & 2914 & 112,4 & 3516 & 1738 & $-50,6$ \\
\hline Castilla La Mancha & 1685 & 1713 & 1,7 & 133 & 385 & 189,8 & 12673 & 4449 & $-64,9$ \\
\hline Murcia & 1024 & 1102 & 7,6 & 86 & 303 & 251,3 & 11865 & 3634 & $-69,4$ \\
\hline Asturias & 1119 & 1086 & $-2,9$ & 221 & 485 & 119,6 & 5065 & 2240 & $-55,8$ \\
\hline La Rioja & 262 & 265 & 0,9 & 50 & 115 & 129,0 & 5222 & 2301 & $-55,9$ \\
\hline Cantabria & 527 & 527 & 0,0 & 98 & 207 & 111,8 & 5382 & 2541 & $-52,8$ \\
\hline País Valenciano & 3745 & 4028 & 7,5 & 665 & 1347 & 102,5 & 5631 & 2990 & $-46,9$ \\
\hline País Vasco & 2148 & 2099 & $-2,3$ & 469 & 999 & 113,3 & 4585 & 2101 & $-54,2$ \\
\hline
\end{tabular}

comparación entre CCAA se realizó con el método apropiado según el tipo de variable (véase pies de tablas 2 y 3). Para cada variable se estimó el cambio porcentual de 1987 a $1997\left[\left(\left(\bar{x}_{97}-\bar{x}_{87}\right) /\right.\right.$ $\left.\bar{x}_{87}\right) \times 100$, donde $\bar{x}$ es la media, porcentaje o total] (véase ref. 11 para la fórmula del error estándar).

\section{Resultados}

Durante el periodo 1987-97, la población española creció un 2,5\%y el número de dentistas creció un $136,1 \%$ produciéndose una reducción de la proporción habitante/dentista del $56,6 \%$ No obstante se obser- varon diferencias relevantes entre las distintas CCAA, tanto en los cocientes habitante/dentista en 1987 y 1997, como en la reducción del mismo (tabla 1).

El porcentaje de población que visitó al dentista en los últimos tres meses creció del 13,5\% en 1987 al $17,2 \%$ en 1997, un incremento relativo y estadísticamente significativo del $26,8 \%$ Si bien hay diferencias significativas entre CCAA en dichos porcentajes, tanto en 1987 como en 1997, no las hay cuando se comparan los cambios porcentuales entre los dos años de estudio (tabla 2). En conjunto, y en 1997 , el $84,4 \%$ de las últimas visitas fueron privadas, lo que signifi- ca un incremento significativo del 9,9\%respecto a 1987, y desapareciendo las diferencias significativas entre CCAA (tabla 2). El número medio de visitas odontológicas privadas en tres meses entre aquellos que visitaron al dentista se situó en 2,22 en 1987 y 2,13 en 1997 , cifras muy estables a lo largo del periodo de estudio, y sin diferencias significativas entre las CCAA (tabla 2).

La tabla 3 recoge el número total de visitas odontológicas privadas realizadas por la población española, pasando de 8,9 millones en 1987 a 12,2 millones en 1997, referido a un periodo de tres meses. Esto indica un incremento del 36,8\%del volumen de 

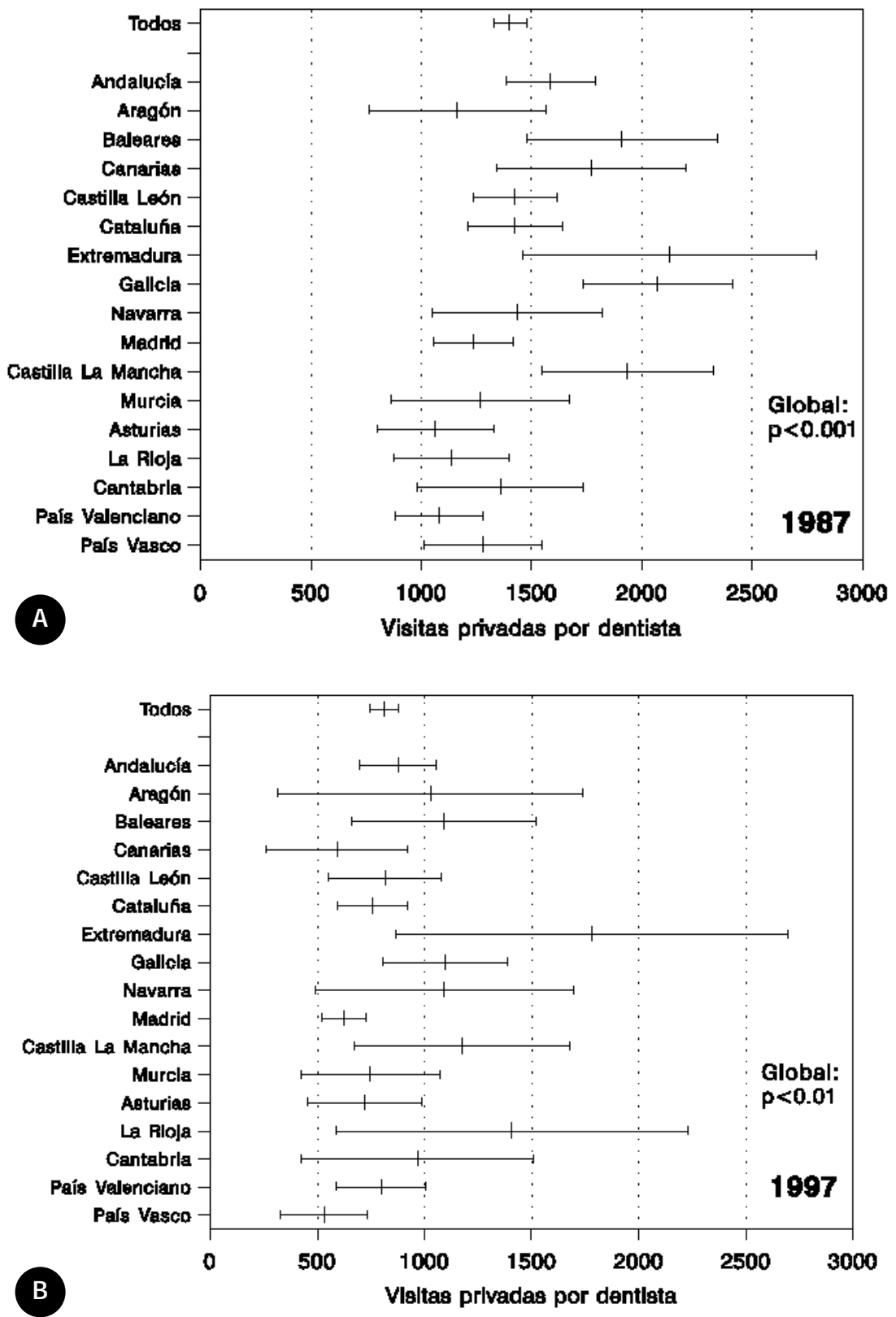

Figura 1. Cargas de trabajo por dentista en España en 1987 (gráfico superior A) y 1997 (gráfico inferior B), medidas como número de visitas privadas atendidas en 3 meses (medias e intervalos de confianza el 95\%).

trabajo total en diez años. Dividiendo esas cifras por el número de dentistas, se obtiene las cargas de trabajo por dentista, que se han reducido un $42,0 \%$ en 10 años. Es destacable que, si bien tanto en 1987 como en 1997 hay diferencias significativas entre CCAA, la significación desaparece cuando se compara entre CCAA la reducción porcentual de cargas de

\begin{tabular}{|lcc}
\hline \multicolumn{3}{|c}{ Tabla 2; Visitas al dentista en } \\
\hline Variable & \multicolumn{2}{c}{$\mathbf{n}^{\mathbf{a}}$} \\
& $\mathbf{1 9 8 7}$ & $\mathbf{1 9 9 7}$ \\
\hline \multicolumn{1}{l}{ Todos } & 39751 & 8383 \\
\hline Comunidad Autónoma & \\
Andalucía & 5980 & 1437 \\
\hline Aragón & 1861 & 231 \\
\hline Baleares & 1201 & 172 \\
\hline Canarias & 1426 & 327 \\
\hline Castilla León & 5269 & 600 \\
\hline Cataluña & 3800 & 1324 \\
\hline Extremadura & 1321 & 236 \\
\hline Galicia & 2849 & 640 \\
\hline Navarra & 1144 & 112 \\
\hline Madrid & 2350 & 1055 \\
\hline Castilla La Mancha & 2995 & 344 \\
\hline Murcia & 1300 & 224 \\
\hline Asturias & 1264 & 267 \\
\hline La Rioja & 1071 & 44 \\
\hline Cantabria & 1156 & 108 \\
\hline País Valenciano & 2626 & 815 \\
\hline País Vasco & 2138 & 447 \\
\hline & & \\
& & \\
\hline
\end{tabular}

trabajo a lo largo de los 10 años de estudio (tabla 3). J unto a la descripción de medias y errores estándar recogidos en la tabla 3 , y dado que son el objetivo central del estudio, las cargas de trabajo se han representado mediante intervalos de confianza en las figuras 1 y 2 , lo que permite un fácil inspección visual, y comparación entre distintas CCAA. Puede observarse, a modo de ejemplo, cómo los dentistas madrileños han mantenido tanto en 1987 como en 1997, menores cargas de trabajo que los extremeños. 
los últimos tres meses en España en 1987 y 1997, estimados a partir de las Encuestas Nacionales de Salud

\begin{tabular}{|c|c|c|c|c|c|c|c|c|}
\hline \multicolumn{3}{|c|}{$\%$ que visita $\pm e^{e}{ }^{b}$} & \multicolumn{3}{|c|}{$\%$ de última visita privada $\pm e^{b}$} & \multicolumn{3}{|c|}{ media de visitas $\pm e^{b}$} \\
\hline 1987 & 1997 & cambio \%c & 1987 & 1997 & cambio \% & 1987 & 1997 & cambio \% \\
\hline $13,5 \pm 0,2$ & $17,2 \pm 0,4$ & $26,8 \pm 3,7 d$ & $76,8 \pm 0,7$ & $84,4 \pm 1,0$ & $9,9 \pm 1,6 \mathrm{~d}$ & $2,22 \pm 0,04$ & $2,13 \pm 0,06$ & $-4,1 \pm 3,2$ \\
\hline$p<0,001^{e}$ & $\mathrm{p}<0,01^{\mathrm{e}}$ & $p=0,262^{e}$ & $\mathrm{p}<0,001^{\mathrm{e}}$ & $p=0,068^{e}$ & $\mathrm{p}<0,001^{\dagger}$ & $\mathrm{p}=0,213^{9}$ & $\mathrm{p}=0,271^{\mathrm{g}}$ & $\mathrm{p}=0,319^{\mathrm{f}}$ \\
\hline $12,6 \pm 0,5$ & $15,2 \pm 0,9$ & $20,9 \pm 8,7^{d}$ & $69,6 \pm 1,8$ & $83,4 \pm 2,5$ & $19,9 \pm 4,8^{d}$ & $2,23 \pm 0,11$ & $2,43 \pm 0,18$ & $9,0 \pm 9,7$ \\
\hline $12,6 \pm 0,1$ & $17,4 \pm 2,4$ & $38,0 \pm 22,0$ & $79,7 \pm 3,0$ & $90,2 \pm 4,6$ & $13,2 \pm 7,2$ & $2,32 \pm 0,25$ & $2,87 \pm 0,89$ & $23,7 \pm 40,8$ \\
\hline $17,2 \pm 1,2$ & $19,4 \pm 3,0$ & $12,8 \pm 19,3$ & $81,3 \pm 3,0$ & $87,6 \pm 5,8$ & $7,7 \pm 8,1$ & $2,32 \pm 0,18$ & $2,53 \pm 0,28$ & $8,9 \pm 14,8$ \\
\hline $13,7 \pm 0,9$ & $11,8 \pm 1,8$ & $-14,1 \pm 14,5$ & $75,6 \pm 3,7$ & $76,5 \pm 6,2$ & $1,2 \pm 9,6$ & $2,14 \pm 0,17$ & $2,13 \pm 0,43$ & $-0,6 \pm 21,7$ \\
\hline $11,2 \pm 0,5$ & $14,7 \pm 1,5$ & $31,2 \pm 14,4^{d}$ & $75,0 \pm 2,0$ & $80,7 \pm 4,2$ & $7,6 \pm 6,3$ & $2,12 \pm 0,10$ & $2,01 \pm 0,23$ & $-5,3 \pm 11,5$ \\
\hline $14,1 \pm 0,7$ & $17,8 \pm 1,0$ & $26,5 \pm 9,6^{d}$ & $83,3 \pm 1,9$ & $83,7 \pm 2,5$ & $0,5 \pm 3,7$ & $2,20 \pm 0,12$ & $1,89 \pm 0,11$ & $-13,8 \pm 6,9^{d}$ \\
\hline $9,9 \pm 1,0$ & $15,7 \pm 2,4$ & $58,7 \pm 28,7^{d}$ & $63,9 \pm 5,1$ & $86,4 \pm 5,7$ & $35,3 \pm 14,0^{d}$ & $2,21 \pm 0,22$ & $2,42 \pm 0,49$ & $9,6 \pm 24,6$ \\
\hline $13,7 \pm 0,6$ & $19,2 \pm 1,6$ & $40,6 \pm 13,5^{d}$ & $78,9 \pm 2,2$ & $87,3 \pm 3,0$ & $10,6 \pm 4,9^{d}$ & $2,08 \pm 0,12$ & $2,21 \pm 0,23$ & $6,1 \pm 12,5$ \\
\hline $13,5 \pm 1,1$ & $19,7 \pm 3,8$ & $46,0 \pm 30,4$ & $78,8 \pm 3,6$ & $90,6 \pm 6,4$ & $15,0 \pm 9,6$ & $2,52 \pm 0,24$ & $2,48 \pm 0,49$ & $-1,6 \pm 2,4$ \\
\hline $17,2 \pm 0,8$ & $21,8 \pm 1,3$ & $26,5 \pm 9,7^{d}$ & $80,8 \pm 2,4$ & $86,0 \pm 2,3$ & $6,5 \pm 4,3$ & $2,51 \pm 0,14$ & $1,99 \pm 0,10$ & $-20,9 \pm 6,0^{d}$ \\
\hline $10,4 \pm 0,6$ & $14,7 \pm 2,1$ & $41,1 \pm 22,1$ & $70,1 \pm 2,9$ & $91,9 \pm 3,9$ & $31,1 \pm 7,9^{d}$ & $2,08 \pm 0,15$ & $1,98 \pm 0,27$ & $-4,6 \pm 14,8$ \\
\hline $10,1 \pm 0,9$ & $15,1 \pm 2,4$ & $49,7 \pm 27,3$ & $60,4 \pm 4,5$ & $81,1 \pm 6,9$ & $34,1 \pm 15,1^{d}$ & $1,77 \pm 0,17$ & $1,75 \pm 0,23$ & $-1,3 \pm 15,9$ \\
\hline $12,6 \pm 1,1$ & $19,1 \pm 2,4$ & $48,3 \pm 22,2^{d}$ & $77,6 \pm 3,2$ & $82,9 \pm 5,2$ & $6,9 \pm 8,0$ & $2,14 \pm 0,19$ & $1,77 \pm 0,21$ & $-17,2 \pm 12,4$ \\
\hline $14,9 \pm 1,1$ & $29,6 \pm 7,0$ & $97,9 \pm 48,8^{d}$ & $77,3 \pm 3,4$ & $100,0 \pm 0,0$ & $29,3 \pm 5,7^{\mathrm{d}}$ & $1,95 \pm 0,15$ & $2,46 \pm 0,45$ & $26,3 \pm 25,1$ \\
\hline $12,8 \pm 1,0$ & $20,4 \pm 3,9$ & $59,5 \pm 32,5$ & $78,1 \pm 3,6$ & $79,7 \pm 8,1$ & $2,1 \pm 11,4$ & $2,56 \pm 0,29$ & $2,33 \pm 0,43$ & $-9,2 \pm 19,7$ \\
\hline $13,0 \pm 0,7$ & $15,2 \pm 1,3$ & $16,8 \pm 11,7$ & $72,8 \pm 2,6$ & $81,3 \pm 3,5$ & $11,7 \pm 6,3$ & $2,02 \pm 0,13$ & $2,26 \pm 0,22$ & $12,0 \pm 12,9$ \\
\hline $15,9 \pm 1,0$ & $17,7 \pm 1,7$ & $11,3 \pm 13,0$ & $83,4 \pm 2,3$ & $81,3 \pm 4,4$ & $-2,5 \pm 6,0$ & $2,12 \pm 0,18$ & $1,69 \pm 0,27$ & $-20,4 \pm 14,3$ \\
\hline
\end{tabular}

\section{Discusión}

En primer lugar se discutirán algunos potenciales sesgos que afectan a este estudio. Respecto a la validez de los censos de dentistas, la falta de colegiación ha sido y es una realidad en España. Normalmente afecta a dentistas (extranjeros o españoles) que han obtenido la titulación fuera de la UE, durante el periodo de instalación de la clínica y la finalización de todos los trámites administrativos y homologación necesarios para la colegiación. En 1989, cuando en términos porcentuales este problema parecía estar en su culmen, se estimó que el porcentaje de dentistas no colegiados se situaba en el $20 \% \%^{2}$. Este problema, en términos porcentuales, está disminuyendo, como ya ha recogido un estudio Delphi ${ }^{3 *}$. Respecto a la validez en la medición de las cargas de trabajo por dentista hay que considerar que en este estudio no se analiza la complejidad de la visita ${ }^{13}$, lo que limitaría la extrapolación a otros contextos profesionales o países. No obstan- te, dado que el objetivo central es valorar los cambios entre 1987 y 1997, la aproximación es válida, como ha sido discutido previamente $e^{2 * *}$.

Los resultados de este estudio indican que se ha producido un incremento de demanda asistencial, pero posiblemente insuficiente con respecto al incremento mucho mayor del número de dentistas. Las previsiones o predicciones (estudio Delphi) hasta el año 2005 indican un incremento en la demanda, pero también en el número de dentistas, endureciendo la presión económica sobre las 


\begin{tabular}{|c|c|c|c|c|c|c|}
\hline \multirow[t]{2}{*}{ Variable } & \multicolumn{3}{|c|}{ Total de visitas (miles) $\pm \mathbf{e e}^{\mathrm{a}}$} & \multicolumn{3}{|c|}{ Visitas/dentista $\pm \mathbf{e e}^{\mathrm{a}}$} \\
\hline & 1987 & 1997 & cambio \% & 1987 & 1997 & cambio \% \\
\hline Todos & $8938 \pm 242$ & $12226 \pm 502$ & $36,8 \pm 6,7^{c}$ & $1402 \pm 38$ & $812 \pm 33$ & $-42,0 \pm 2,8^{c}$ \\
\hline $\begin{array}{l}\text { Comunidad Autónoma } \\
\text { Andalucía }\end{array}$ & $1320 \pm 86$ & $2109 \pm 218$ & $59,8 \pm 19,5^{c}$ & $1588 \pm 103$ & $876 \pm 90$ & $-44,8 \pm 6,7^{c}$ \\
\hline Aragón & $268 \pm 47$ & $473 \pm 167$ & $76,5 \pm 69,5$ & $1164 \pm 204$ & $1029 \pm 362$ & $-11,6 \pm 34,8$ \\
\hline Baleares & $230 \pm 27$ & $360 \pm 72$ & $56,7 \pm 36,3$ & $1912 \pm 221$ & $1091 \pm 218$ & $-42,9 \pm 13,2^{c}$ \\
\hline Canarias & $335 \pm 41$ & $293 \pm 84$ & $-11,7 \pm 27,3$ & $1774 \pm 218$ & $594 \pm 168$ & $-66,5 \pm 10,3^{c}$ \\
\hline Castilla León & $472 \pm 32$ & $651 \pm 108$ & $37,8 \pm 24,7$ & $1426 \pm 97$ & $816 \pm 135$ & $-42,7 \pm 10,3^{c}$ \\
\hline Cataluña & $1575 \pm 122$ & $1770 \pm 194$ & $12,4 \pm 15,1$ & $1427 \pm 110$ & $757 \pm 82$ & $-47,0 \pm 7,1^{c}$ \\
\hline Extremadura & $153 \pm 24$ & $379 \pm 99$ & $147,6 \pm 75,9$ & $2127 \pm 339$ & $1780 \pm 466$ & $-16,3 \pm 25,7$ \\
\hline Galicia & $635 \pm 53$ & $1126 \pm 154$ & $77,3 \pm 28,4^{c}$ & $2071 \pm 173$ & $1097 \pm 146$ & $-47,0 \pm 8,5^{c}$ \\
\hline Navarra & $138 \pm 19$ & $243 \pm 68$ & $75,3 \pm 55,0$ & $1437 \pm 197$ & $1094 \pm 309$ & $-23,8 \pm 23,9$ \\
\hline Madrid & $1699 \pm 127$ & $1817 \pm 153$ & $7,0 \pm 12,0$ & $1238 \pm 92$ & $623 \pm 52$ & $-49,6 \pm 5,7^{c}$ \\
\hline Castilla La Mancha & $257 \pm 26$ & $453 \pm 99$ & $75,9 \pm 42,4$ & $1936 \pm 198$ & $1175 \pm 256$ & $-39,3 \pm 14,6^{c}$ \\
\hline Murcia & $110 \pm 18$ & $227 \pm 50$ & $107,1 \pm 56,7$ & $1269 \pm 206$ & $748 \pm 164$ & $-41,1 \pm 16,1^{c}$ \\
\hline Asturias & $235 \pm 30$ & $351 \pm 66$ & $49,0 \pm 33,9$ & $1066 \pm 136$ & $723 \pm 136$ & $-32,2 \pm 15,5^{c}$ \\
\hline La Rioja & $57 \pm 7$ & $162 \pm 48$ & $183,5 \pm 90,7^{c}$ & $1136 \pm 133$ & $1407 \pm 419$ & $23,8 \pm 39,6$ \\
\hline Cantabria & $133 \pm 19$ & $201 \pm 57$ & $51,0 \pm 48,2$ & $1359 \pm 192$ & $969 \pm 277$ & $-28,7 \pm 22,7$ \\
\hline País Valenciano & $719 \pm 64$ & $1076 \pm 144$ & $49,6 \pm 24,4^{c}$ & $1081 \pm 101$ & $798 \pm 106$ & $-26,2 \pm 12,0^{c}$ \\
\hline País Vasco & $601 \pm 64$ & $533 \pm 103$ & $-11,3 \pm 19,6$ & $1282 \pm 137$ & $533 \pm 103$ & $-58,4 \pm 9,2^{c}$ \\
\hline
\end{tabular}

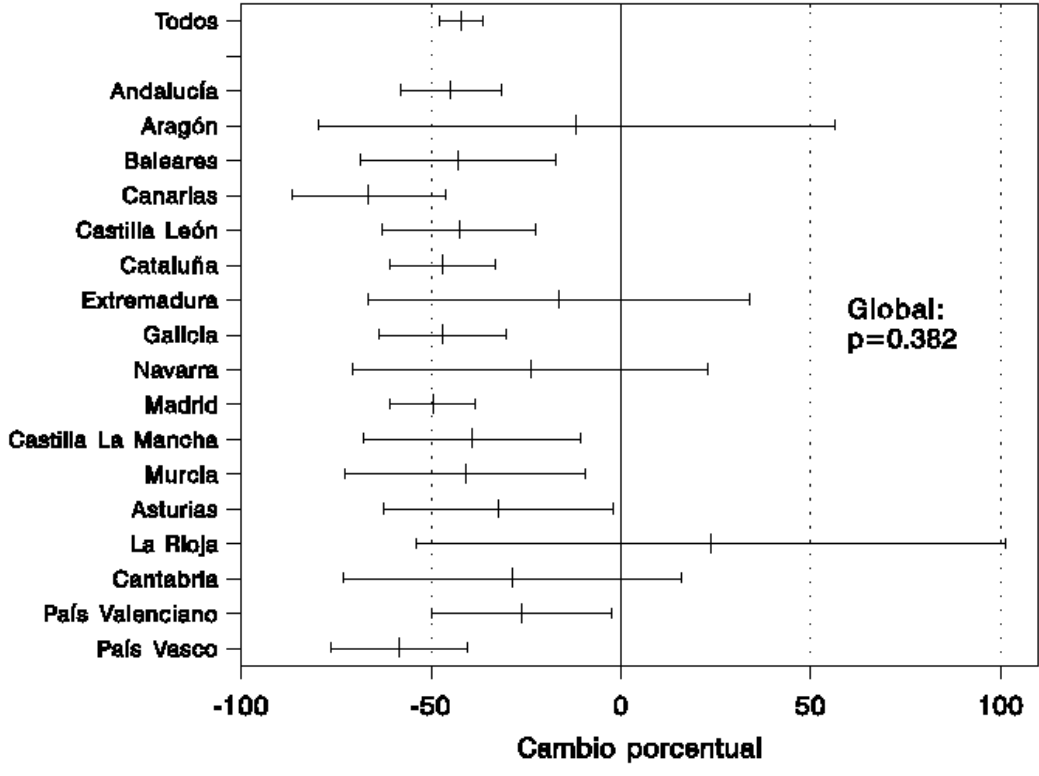

clínicas privadas ${ }^{3 *}$, lo que indicaría que se continuará con el desequilibrio entre la oferta y la demanda. El porcentaje de visitas privadas se ha incrementado, y se deriva de la estabilidad en el número de dentistas públicos, en comparación con el mayor incremento del número de privados. Las diferencias geográficas existen en España, no sólo en el porcentaje de población que acude al dentista, sino también en cuanto a

Figura 2. Cambio porcentual entre $1987 y$ 1997 de las cargas de trabajo por dentista en España (porcentajes medios e intervalos de confianza al 95\%). 
las cargas de trabajo por dentista. Estas diferencias se han mantenido en general durante los diez años de estudio, en un periodo de máximo incremento de profesionales.

Una posible tentación sería concluir que el incremento de dentistas ha sido la razón principal del incremento en la demanda odontológica. No obstante, debe tenerse presente que la demanda depende de un complejo escenario de factores. Uno de los modelos más utilizados para explicar la utilización de servicios de salud es el de Anderson ${ }^{14^{*}}$, que incluye variables predisponentes (edad, sexo, número de dientes presentes, etc.), facilitadores (accesibilidad a los servicios de salud, precio, etc) y de necesidad (dolor, estética, etc.). Por tanto, la accesibilidad es sólo una pequeña parte del total de factores (para una revisión de modelos de demanda véase ref. 15). También destaca que, a pesar de la reducción de las cargas de trabajo por dentista en todas las CCAA, no se ha producido un incremento en el número de visitas de aquellos pacientes que acuden al dentista. Esto, en principio, indicaría que los dentistas no están induciendo demanda, aunque sería necesario conocer la complejidad y número de servicios prestados en cada visita para realizar esta afirmación más concluyentemente, algo no posible con este estudio.

El rápido incremento en el número de dentistas debería conducir al reconocimiento de la necesidad de una coordinación y monitorización a nivel nacional de variables de interés como son el número de profesionales, el número de Facultades de Odontología, de Títulos extranjeros convalidados, la productividad y provisión de servicios, etc., como se ha realizado para Cataluña ${ }^{16^{*}}$. Dado que las cargas de trabajo por dentista continuarán disminuyendo en Espa- ñ $a^{3^{*}}$, y la población española aún acumula numerosas necesidades de tratamiento junto a bajas tasas de visita al dentista, se hace necesario una estrategia activa de mercado, como ha sido demostrada en otros países ${ }^{17}$. Habría que informar adecuadamente a los futuros profesionales sobre los desequilibrios geográficos en las cargas de trabajo.

Las conclusiones de este estudio son: a) Tanto en 1987 como en 1997 hay variaciones en el porcentaje de población que ha acudido al dentista en los últimos tres meses entre CCAA, y b) Respecto a las cargas de trabajo por dentista, medidas como número de visitas privadas atendidas en los últimos tres meses, tanto en 1987 como en 1997 hay diferencias significativas entre CCAA observándose una reducción significativa en el conjunto del país del 42,0\%(ee=2,8\%) y sin diferencias estadísticamente significativas entre las distintas CCAA.

\section{Agradecimientos}

Al Ministerio de Sanidad y Consumo, que durante varios años ha facilitadolas bases de datos de las Encuestas Nacionales de Salud.

\section{Bibliografía recomendada}

Para profundizar en la lectura de este tema, el/los autor/es considera/an interesantes los artículos que aparecen señalados del siguiente modo: *de interés **de especial interés.

1. Noguerol-Rodríguez B, Follana-Murcia M, Ugarte-Ozcoidi M. Demografía de la Profesión Dental Española 1998. RCOE 1999;4 ( ${ }^{\circ}$ especial):17-232.

$2 * *$. Bravo M. Private dental visits per dentist in
Spain from 1987 to 1997 . An analysis from the Spanish National Health Surveys. Community Dent Oral Epidemiol 2002;30:321-8. Estudio que utiliza las ENSs de 1987, 1993, 1995 y 1997. Analiza, a nivel global español, la evolución de las cargas de trabajo por dentista, y está en la misma línea de investigación que el estudio presente.

3*. Libro Blanco. Odonto-estomatología 2005. Barcelona: Lácer, S.A., 1996. 
Proyecto liderado por el Consejo General de Odontólogos y Estomatólogos con dos partes: una encuesta a población general sobre uso de servicios odontológicos, y un estudio Delphi realizados por expertos y sobre el futuro de la profesión odontológica.

4. Noguerol-Rodríguez B, Llodra-Calvo JC, SiciliaFelechosa A, Follana-Murcia M. La salud bucodental en España. 1994. Antecedentes y perspectivas de futuro. Madrid: Ediciones Avances, 1995.

5. Llodra-Calvo JC, Bravo-Pérez M, Cortés-Martinicorena FJ. Encuesta de salud oral de España (2000). RCOE 2002;7 (Monográfico):1963.

6. Bravo M. Age-Period-Cohort analysis of dentist use in Spain from 1987 to 1997. An analysis from the Spanish National Health Surveys. Eur J Oral Sci 2001;109:149-54.

7. Ministerio de Sanidad y Consumo. Encuesta Nacional de Salud. Madrid: Ministerio de Sanidad y Consumo, 1989.

8. Ministerio de Sanidad y Consumo. Encuesta
Nacional de Salud 1997. Madrid: Ministerio de Sanidad y Consumo, 1999.

9. Shah BV, BarnWell BG, Bieler GS. SUDAAN software for the statistical analysis of correlated data. User's manual. Release 7.0. Research Triangle Park, NC: Research Triangle Institute, 1996.

10. DerSimonian R, Laird N. Meta-analysis in clinical trials. Control Clin Trials 1986;7: 177-88.

11. Dubey SO, Lehnhoff RW, Radike AW. A statistical confidence interval for true percent reduction in caries-incidence studies. J Dent Res 1965;44:921-3.

12. Castaño-Seiquer A, Cordero-Bulnes MA, Fernández-Ollero A, González-Serrano A. Análisis de los recursos humanos odontoestomatológicos en la provincia de Cádiz. Rev Andal Odontol Estomatol 1991;1:37-44.

13. Council on Dental Health, Bureau of Economic Research and Statistics. Study of relative values of dental services. J Am Dent Assoc 1968;76:117-22.
14*. Andersen R, Newman JF. Societal and individual determinants of medical care utilization in the United States. Milbank Mem Mund Q 1973;51:95-124.

Constituye una referencia obligada sobre los factores asociados con la demanda y utilización de servicios sanitarios.

15. Chen M, Andersen RM, Barmes DE, Leclercq $\mathrm{MH}$, Lyttle CS. Comparing oral health care systems. A second international collaborative study. Geneva: World Health Organization, 1997.

16*. Cuenca-Sala E, Gómez-Jiménez A, Casals-Peidró E, Subirá-Pifarré C. La Odontología en Cataluña 2002. Barcelona: Colegi Oficial d'Odontolegs i Estomatolegs de Catalunya, 2002.

Restringido a Cataluña, constituye un análisis de la situación profesional odontológica desde distintas perspectivas, así como recomendaciones futuras.

17. Anderson RJ, Morgan JD. Marketing dentistry: a pilot study in Dudley. Community Dent Health 1992;9 (Suppl 1):1-220. 